Chinese Journal of Organic Chemistry

\title{
Aspernigerin 类似物的合成及生物活性研究
}

\author{
吴清来 ${ }^{a}$ 李永强 $b \quad$ 杨新玲 ${ }^{a}$ 凌 云 $*, a$ \\ ( ${ }^{a}$ 中国农业大学理学院应用化学系 农业部农药化学与应用重点开放实验室 北京 100193) \\ ( ${ }^{b}$ 南开大学元素有机化学国家重点实验室 天津 300071)
}

\begin{abstract}
摘要 以天然产物 Aspernigerin 为先导, 将 Aspernigerin 结构中的一个 1,2,3,4-四氢喹啉基团以芳胺类化合物替代, 设计 合成了 15 个未见文献报道的 Aspernigerin 类似物, 结构均经过 ${ }^{1} \mathrm{H}$ NMR, IR 和元素分析确证. 初步生物活性测试表明, 大部分目标化合物均表现出了一定的杀虫和杀菌活性, 其中化合物 $2 \mathrm{n}$ 在 $200 \mu \mathrm{g} / \mathrm{mL}$ 的浓度下对小菜蛾仍表现出 $100 \%$ 的杀虫活性，优于先导 Aspernigerin 和对照药剂噻虫嗪; 化合物 $\mathbf{2 d}, \mathbf{2 e}, \mathbf{2 j}, \mathbf{2 k}$ 表现出对黄瓜灰霉病优于先导 Aspernigerin 的抑制活性.
\end{abstract}

关键词 Aspernigerin; 类似物; 合成; 1,2,3,4-四氢喹啉; 芳胺; 生物活性

\section{Synthesis and Bioactivity of Aspernigerin Analogues}

\author{
Wu, Qinglai ${ }^{a}$ \\ Li, Yongqiang ${ }^{b}$ \\ Yang, Xinling ${ }^{a}$ \\ Ling, Yun ${ }^{*, a}$ \\ ( ${ }^{a}$ Key Laboratory of Pesticide Chemistry and Application, Ministry of Agriculture, Department of Applied Chemistry, \\ College of Science, China Agricultural University, Beijing 100193) \\ ( ${ }^{b}$ State Key Laboratory of Elemento-Organic Chemistry, Nankai University, Tianjin 300071)
}

\begin{abstract}
Using natural product aspernigerin as the lead compound, fifteen new compounds were designed and synthesized by replaced one 1,2,3,4-tetrahydroquinoline group of aspernigerin with aromatic amine. Those structures were confirmed by ${ }^{1} \mathrm{H}$ NMR, IR spectra and elemental analysis. The preliminary bioassay showed that most of the compounds exhibited certain fungicidal activities and insecticidal activities. In particular, compound $\mathbf{2 n}$ showed the most potent bioactivity against Plutella xylostella with the rate of $100 \%$ at the concentration of $200 \mu \mathrm{g} / \mathrm{mL}$, better than aspernigerin and commercial insecticide thiamethoxam, and compounds $\mathbf{2 d}, \mathbf{2 e}, \mathbf{2} \mathbf{j}, \mathbf{2} \mathbf{k}$ showed higher inhibition effects against Botrytis cinerea than aspernigerin.
\end{abstract}

Keywords aspernigerin; analogues; synthesis; 1,2,3,4-tetrahydroquinoline; aromatic amine; bioactivity

Aspernigerin 是由申丽等 ${ }^{[1,2]}$ 于 2006 年首次从狗牙 根内生菌 Aspergillus niger IFB-E003 的固体培养物提取 液中分离得到的, 其结构经确证发现是一个骨架新颖的 生物碱类次生代谢产物. 进一步的体外细胞毒活性测试 发现, Aspernigerin 对人的口腔表皮样癌细胞 KB、宫颈 癌细胞 Hela、结肠癌细胞 SW1116 表现出较强的细胞毒 活性, 其 $\mathrm{IC}_{50}$ 值分别为 22,46 和 $35 \mu \mathrm{mol} / \mathrm{L}$. 阳性对照 5氟尿嘧啶 (5-FU) 对这三株癌细胞株的 $\mathrm{IC}_{50}$ 值分别为 14 , 115 和 $42 \mu \mathrm{mol} / \mathrm{L}$.

本课题组在前期的工作中, 首次对 Aspernigerin 进 行农药生物活性测试, 结果表明, Aspernigerin 具有一定 的杀菌、杀虫活性, 尤其是对小菜蛾表现出较好的生物
活性，在 600 和 $200 \mu \mathrm{g} / \mathrm{mL}$ 的浓度下对小菜蛾的杀虫活 性分别为 $100 \%$ 和 $75 \%$, 略低于对照药剂噻虫嗪. 因此, Aspernigerin 是一个具有优良活性的潜在药物分子或先 导化合物.<smiles>O=C(CN1CCCc2ccccc21)N1CCN(CC(=O)N2CCCc3ccccc32)CC1</smiles>

本工作以 Aspernigerin 为先导化合物进行结构改造 与修饰, 将 Aspernigerin 结构中的一个 1,2,3,4-四氢喹啉

\footnotetext{
*E-mail:1yun@cau.edu.cn

Received October 9, 2011; revised November 16, 2011; published online December 9, 2011. 2010CB126104).

国家自然科学基金(No. 21072222)和国家 973 计划(No. 2010CB126104)资助项目.
}

Project supported by the National Natural Science Foundation of China (No. 21072222) and the National Basic Research Program of China (973 Program, No. 
基团用胺类化合物替代，设计合成了 15 个 Aspernigerin 类似物，以期发现具有更好生物活性的化合物，并为下 一步开展 Aspernigerin 类化合物的构效关系, 指明该类 结构改造与修饰的方向奠定了基础. 目标化合物的合成 如 Scheme 1 所示.
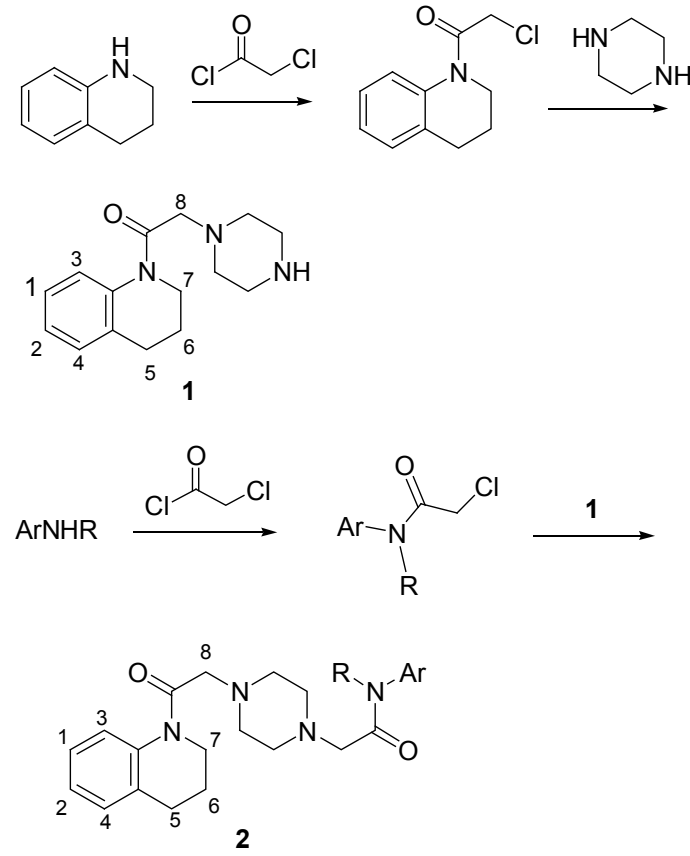

2a: $\mathrm{Ar}=\mathrm{C}_{6} \mathrm{H}_{5}, \mathrm{R}=\mathrm{H} ; \mathbf{2 b}: \mathrm{Ar}=4-\mathrm{F}-3-\mathrm{NO}_{2} \mathrm{C}_{6} \mathrm{H}_{3}, \mathrm{R}=\mathrm{H} ; \mathbf{2 c}: \mathrm{Ar}=$ $\mathrm{C}_{6} \mathrm{H}_{5} \mathrm{CHCH}_{3}, \mathrm{R}=\mathrm{H}$; 2d: $\mathrm{Ar}=4-\mathrm{NO}_{2}-3-\mathrm{CF}_{3} \mathrm{C}_{6} \mathrm{H}_{3}, \mathrm{R}=\mathrm{H} ; \mathbf{2 e}: \mathrm{Ar}=$ 2- $\mathrm{NO}_{2} \mathrm{C}_{6} \mathrm{H}_{4}, \mathrm{R}=\mathrm{H} ; \mathbf{2 f}: \mathrm{Ar}=2,6-\left(\mathrm{CH}_{3}\right)_{2} \mathrm{C}_{6} \mathrm{H}_{3}, \mathrm{R}=\mathrm{H} ; \mathbf{2 g}: \mathrm{Ar}=$ 4,6- $\left(\mathrm{CH}_{3} \mathrm{O}\right)_{2} \mathrm{C}_{4} \mathrm{HN}_{2}, \mathrm{R}=\mathrm{H} ; \mathbf{2 h}: \mathrm{Ar}=4-\mathrm{NO}_{2}-2-\mathrm{CF}_{3} \mathrm{C}_{6} \mathrm{H}_{3}, \mathrm{R}=\mathrm{H} ; \mathbf{2 i}$ : $\mathrm{Ar}=\mathrm{C}_{6} \mathrm{H}_{5}, \mathrm{R}=\mathrm{C}_{6} \mathrm{H}_{5} ; \quad 2 \mathrm{j}: \mathrm{Ar}=2,6-\mathrm{F}_{2} \mathrm{C}_{6} \mathrm{H}_{3}, \mathrm{R}=\mathrm{H} ; \mathbf{2 k}: \mathrm{Ar}=$ 4- $\mathrm{CH}_{3} \mathrm{OC}_{6} \mathrm{H}_{4}, \mathrm{R}=\mathrm{H} ; \quad 2 \mathrm{I}: \mathrm{Ar}=4-\mathrm{CH}_{3} \mathrm{C}_{6} \mathrm{H}_{4}, \mathrm{R}=\mathrm{H} ; \quad 2 \mathrm{~m}: \mathrm{Ar}=$ 4- $\mathrm{CF}_{3}-2-\mathrm{NO}_{2} \mathrm{C}_{6} \mathrm{H}_{3}, \mathrm{R}=\mathrm{H} ; \mathbf{2 n}: \mathrm{Ar}=3-\mathrm{NO}_{2} \mathrm{C}_{6} \mathrm{H}_{4}, \mathrm{R}=\mathrm{H} ; \mathbf{2 0}: \mathrm{Ar}=\mathrm{C}_{6} \mathrm{H}_{5}$, $\mathrm{R}=\mathrm{CH}_{3}$

\section{Scheme 1}

\section{1 实验部分}

\section{1 仪器及试剂}

熔点测定仪: Yanagimoto MFG，温度计未校正; 核 磁共振仪: Bruker Avance DPX300, 以 TMS 为内标, 氛 代氯仿为溶剂; 红外光谱仪: Shimadzu IR-435(采用液膜 法); 元素分析仪: ST-Carloerba.Co(采用 Flash EA 1112 法); HPLC: 岛津 LC-10A PDA, 色谱柱: Agilent Extend $\mathrm{C} 18,250 \mathrm{~mm} \times 4.6 \mathrm{~mm}, 5 \mu \mathrm{m}$.

所用的药品均为市售的分析纯或化学纯, 除特别注 明外, 未经进一步处理.

\section{2 合成实验}

\subsection{1 $N$-氯乙酰-1,2,3,4-四氢喹啉的合成}

于 $500 \mathrm{~mL}$ 三口烧瓶中, 加入 $26.6 \mathrm{~g}(0.2 \mathrm{~mol})$ 1,2,3,4-四氢喹啉和 $300 \mathrm{~mL}$ 二氯甲烷, 冰盐浴降温至 $-5 \sim 0{ }^{\circ} \mathrm{C}$. 缓慢滴加 $25.0 \mathrm{~g}(0.22 \mathrm{~mol})$ 氯乙酰氯和 100
$\mathrm{mL}$ 二氯甲烷配成的溶液, 滴加完毕, 搅拌反应 $10 \mathrm{~min}$, 然后缓慢滴加 $21.2 \mathrm{~g}(0.21 \mathrm{~mol})$ 三乙胺, 滴加完毕后, 保 温于 $-5 \sim 5{ }^{\circ} \mathrm{C}$ 反应 $5 \sim 8 \mathrm{~h}$, 薄层硅胶色谱板监控确定 反应终点. 反应完全后，加入 $50 \mathrm{~mL} 5 \%$ 的盐酸水溶液, 摚拌 $30 \mathrm{~min}$, 静置分层，分出有机层，再用 $100 \mathrm{~mL} \mathrm{5 \%}$ 的盐酸水溶液洗涤一遍，分出有机层，用无水硫酸钠干 燥, 过滤, 滤液先常压蒸馏脱出溶剂, 然后, 油泵减压 蒸馏, 收集 $162 \sim 164{ }^{\circ} \mathrm{C} / 130 \mathrm{~Pa}$ 的馏分 $38.8 \mathrm{~g}$, 即为 $N$ 氯乙酰-1,2,3,4-四氢喹啉，收率 $92.8 \%$. m.p. 52 53 ${ }^{\circ} \mathrm{C}$ (文献值 $\left.{ }^{[3]}: 53 \sim 54{ }^{\circ} \mathrm{C}\right) ;{ }^{1} \mathrm{H}$ NMR $\left(300 \mathrm{MHz}, \mathrm{CDCl}_{3}\right) \delta$ : $1.88 \sim 1.97\left(\mathrm{~m}, 2 \mathrm{H}, \mathrm{CH}_{2} \mathrm{CH}_{2} \mathrm{CH}_{2}\right), 2.71(\mathrm{t}, J=6.6 \mathrm{~Hz}, 2 \mathrm{H}$, $\left.\mathrm{ArCH}_{2}\right), 3.71\left(\mathrm{t}, J=6.3 \mathrm{~Hz}, 2 \mathrm{H}, \mathrm{NCH}_{2}\right), 4.55(\mathrm{~s}, 2 \mathrm{H}$, $\mathrm{CH}_{2} \mathrm{Cl}$ ); EI-MS, $m / z$ (\%): $209\left(\mathrm{M}^{+}, 48\right), 174$ (42), 132 (100), 77 (26).

1.2.2 $N$-(1,2,3,4-四氢喹啉基- $N$-甲酰甲基)哌嗪的合 成

于 $500 \mathrm{~mL}$ 三口烧瓶中, 加入 $36.2 \mathrm{~g}(0.42 \mathrm{~mol})$ 哌嗪、 $42.0 \mathrm{~g}(0.3 \mathrm{~mol})$ 无水碳酸钾和 $100 \mathrm{~mL} N, \mathrm{~N}$-二甲基甲酰 胺, 搅拌, 加热保温于 $80 \sim 100{ }^{\circ} \mathrm{C}$ 之间. 滴加 $44.0 \mathrm{~g}$ (0.21 mol) $N$-氯乙酰-1,2,3,4-四氢喹啉和 $50 \mathrm{~mL} N, N$-二 甲基甲酰胺配成的溶液，滴加完毕后，保温于 $80 \sim 100$ ${ }^{\circ} \mathrm{C}$ 反应 4 6 h, 薄层硅胶色谱板监控确定反应终点. 反 应完全后，降温至室温，抽滤，除去固体盐类物质，滤 液水泵减压蒸馏, 蒸出 $N, N$-二甲基甲酰胺, 残留物加入 $100 \mathrm{~mL}$ 三氯甲烷, 加热回流 $10 \mathrm{~min}$ 使溶解完全, 然后 降至室温，于铺有硅胶的砂芯漏斗中抽滤，然后用乙酸 甲酯淋洗硅胶, 滤液脱溶剂, 得到 $N-(1,2,3,4$-四氢喹啉 基- $N$-甲酰甲基)哌嗪粗品. 粗品进一步柱层析 [ $V$ ( 乙酸甲 酯) : $V$ (石油醚 $)=1 ： 2$ ]纯化得 $N-(1,2,3,4$-四氢喹啉基$N$-甲酰甲基)哌嗪 $39.3 \mathrm{~g}$, 收率 72.0\%, HPLC 分析归一含 量为 $\geqslant 98 \%$. ${ }^{1} \mathrm{H}$ NMR $\left(300 \mathrm{MHz}, \mathrm{CDCl}_{3}\right) \delta: 1.86 \sim 1.95$ (m, 2H, 6-H), 2.62 (brs, 4H, pip-H), 2.65 (t, $J=10.2 \mathrm{~Hz}$, 2H, 5-H), 2.95 (brs, 4H, pip-H), 3.27 (s, 2H, 8-H), 6.42 (s, $1 \mathrm{H}, \mathrm{NH}) 7.06 \sim 7.14(\mathrm{~m}, 3 \mathrm{H}, \mathrm{ArH}), 7.62$ (brs, 1H, 3-H); ESI-MS $m / z$ (\%): $260\left(\mathrm{M}^{+}, 100\right)$. Anal. calcd for $\mathrm{C}_{15} \mathrm{H}_{21} \mathrm{~N}_{3} \mathrm{O}$ : C 69.47, H 8.16, N 16.20; found C $69.28, \mathrm{H}$ 8.18, N 16.31 .

\subsection{3 氯乙酰取代芳胺的合成}

氯乙酰取代芳胺参照文献[4]方法合成. 于 $500 \mathrm{~mL}$ 三口烧瓶中, 加入 $0.1 \mathrm{~mol}$ 取代芳胺和 $200 \mathrm{~mL}$ 二氯甲 烷, 冰盐浴降温至 $-5 \sim 0{ }^{\circ} \mathrm{C}$. 缓慢滴加 $12.5 \mathrm{~g}(0.11$ $\mathrm{mol}$ )氯乙酰氯和 $100 \mathrm{~mL}$ 二氯甲烷配成的溶液, 滴加完 毕, 搅拌反应 $10 \mathrm{~min}$, 然后缓慢滴加 $11.0 \mathrm{~g}(0.11 \mathrm{~mol})$ 三 乙胺, 滴加完毕后, 保温于 $-5 \sim 5{ }^{\circ} \mathrm{C}$ 反应 $5 \sim 8 \mathrm{~h}$, 薄层 硅胶色谱板监控确定反应终点. 反应完全后，加入 50 
$\mathrm{mL} \mathrm{5 \%}$ 的盐酸水溶液, 搅拌 $30 \mathrm{~min}$, 静置分层, 分出有

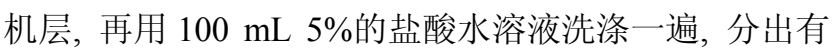
机层, 用无水硫酸钠干燥、过滤, 滤液脱溶, 用甲醇重结 晶，过滤烘干，得氯乙酰基取代芳胺化合物.

1.2.4 目标化合物 $N$ - $(1,2,3,4$-四氢喹啉基- $N$-甲酰甲 基)- $N^{\prime}$-(取代芳胺基- $N$-甲酰甲基)哌嗪的合成

以目标化合物 $\mathbf{2 a}$ 为例: 于 $500 \mathrm{~mL}$ 三口烧瓶中, 加 入 $N$-(1,2,3,4-四氢喹啉基- $N$-甲酰甲基)哌嗪 $1.3 \mathrm{~g}$ (0.01 $\mathrm{mol}) 、$ 三乙胺 $1.2 \mathrm{~g}(0.012 \mathrm{~mol})$ 和 $50 \mathrm{~mL}$ 三氯甲烷, 加热
至回流，缓慢滴加 $0.012 \mathrm{~mol}$ 氯乙酰取代芳胺和 $50 \mathrm{~mL}$ 三氯甲烷配成的溶液，滴加完毕，回流反应 $2 \sim 3 \mathrm{~h}$, 薄 层硅胶色谱板监控确定反应终点. 反应完全后，降温至 室温，于铺有硅胶的砂芯漏斗中抽滤，然后用三氯甲烷 淋洗硅胶, 滤液脱溶剂得目标产物粗品. 粗品进一步柱 层析 $[V$ (乙酸甲酯) $: V$ (石油醚 $)=1: 9$ ] 纯化得目标产物 纯品, HPLC 分析归一含量为 $\geqslant 99 \%$. 采用同样的方法合 成了化合物 $\mathbf{2 b} \sim \mathbf{2 0}$, 化合物的理化、元素分析、核磁和 IR 数据分别见表 1 和表 2 .

表 1 化合物 $\mathbf{2 a} \sim \mathbf{2 0}$ 的元素分析及物理性质数据

Table 1 Physical data and elemental analysis of compounds $\mathbf{2 a} \sim \mathbf{2 0}$

\begin{tabular}{|c|c|c|c|c|c|c|c|}
\hline \multirow{2}{*}{ Compd. } & \multirow{2}{*}{ Formula } & \multirow{2}{*}{ m.p. $/{ }^{\circ} \mathrm{C}$} & \multirow{2}{*}{ Appearance } & \multirow{2}{*}{ Yield/\% } & \multicolumn{3}{|c|}{ Elemental analysis (calcd.) } \\
\hline & & & & & $\mathrm{C}$ & $\mathrm{H}$ & $\mathrm{N}$ \\
\hline $2 a$ & $\mathrm{C}_{23} \mathrm{H}_{28} \mathrm{~N}_{4} \mathrm{O}_{2}$ & $43 \sim 45$ & Yellow solid & 69.5 & $70.12(70.38)$ & $7.18(7.19)$ & $14.31(14.27)$ \\
\hline $2 \mathrm{~b}$ & $\mathrm{C}_{23} \mathrm{H}_{26} \mathrm{FN}_{5} \mathrm{O}_{4}$ & $134 \sim 135$ & Yellow solid & 66.0 & $60.46(60.65)$ & $5.87(5.75)$ & $15.08(15.38)$ \\
\hline $2 \mathrm{c}$ & $\mathrm{C}_{25} \mathrm{H}_{32} \mathrm{~N}_{4} \mathrm{O}_{2}$ & $36 \sim 38$ & Yellow solid & 75.8 & $71.43(71.40)$ & $7.76(7.67)$ & $13.22(13.32)$ \\
\hline 2d & $\mathrm{C}_{24} \mathrm{H}_{26} \mathrm{~F}_{3} \mathrm{~N}_{5} \mathrm{O}_{4}$ & $60 \sim 62$ & Yellow solid & 71.6 & $56.91(57.03)$ & $5.26(5.18)$ & $13.76(13.85)$ \\
\hline $2 e$ & $\mathrm{C}_{23} \mathrm{H}_{27} \mathrm{~N}_{5} \mathrm{O}_{4}$ & $126 \sim 127$ & Yellow solid & 66.8 & $63.19(63.14)$ & $6.18(6.22)$ & $15.88(16.01)$ \\
\hline $2 f$ & $\mathrm{C}_{25} \mathrm{H}_{32} \mathrm{~N}_{4} \mathrm{O}_{2}$ & $38 \sim 39$ & Yellow solid & 68.6 & $71.18(71.40)$ & 7.79 (7.67) & $13.45(13.32)$ \\
\hline $2 \mathrm{~g}$ & $\mathrm{C}_{23} \mathrm{H}_{30} \mathrm{~N}_{6} \mathrm{O}_{4}$ & $34 \sim 35$ & Yellow solid & 70.5 & $60.55(60.78)$ & $6.46(6.65)$ & $18.81(18.49)$ \\
\hline $2 \mathrm{~h}$ & $\mathrm{C}_{24} \mathrm{H}_{26} \mathrm{~F}_{3} \mathrm{~N}_{5} \mathrm{O}_{4}$ & $152 \sim 154$ & Brown solid & 78.2 & $56.98(57.03)$ & $5.24(5.18)$ & $13.61(13.85)$ \\
\hline $2 \mathbf{i}$ & $\mathrm{C}_{29} \mathrm{H}_{32} \mathrm{~N}_{4} \mathrm{O}_{2}$ & $35 \sim 36$ & Yellow solid & 68.0 & $74.21(74.33)$ & $6.94(6.88)$ & $11.83(11.96)$ \\
\hline $2 \mathbf{j}$ & $\mathrm{C}_{23} \mathrm{H}_{26} \mathrm{~F}_{2} \mathrm{~N}_{4} \mathrm{O}_{2}$ & $115 \sim 117$ & White solid & 66.5 & $64.20(64.47)$ & $6.12(6.18)$ & $12.89(13.08)$ \\
\hline $2 \mathrm{k}$ & $\mathrm{C}_{24} \mathrm{H}_{30} \mathrm{~N}_{4} \mathrm{O}_{3}$ & $53 \sim 55$ & Yellow solid & 73.0 & $68.43(68.22)$ & $7.18(7.16)$ & $13.09(13.26)$ \\
\hline 21 & $\mathrm{C}_{24} \mathrm{H}_{30} \mathrm{~N}_{4} \mathrm{O}_{2}$ & $47 \sim 49$ & Yellow solid & 82.6 & $70.68(70.91)$ & $7.65(7.44)$ & $13.55(13.78)$ \\
\hline $2 m$ & $\mathrm{C}_{24} \mathrm{H}_{26} \mathrm{~F}_{3} \mathrm{~N}_{5} \mathrm{O}_{4}$ & $110 \sim 112$ & White solid & 68.8 & $57.05(57.03)$ & $5.31(5.18)$ & $13.72(13.85)$ \\
\hline $2 n$ & $\mathrm{C}_{23} \mathrm{H}_{27} \mathrm{~N}_{5} \mathrm{O}_{4}$ & $115 \sim 117$ & White solid & 65.5 & $63.22(63.14)$ & $6.20(6.22)$ & $15.99(16.01)$ \\
\hline 20 & $\mathrm{C}_{24} \mathrm{H}_{30} \mathrm{~N}_{4} \mathrm{O}_{2}$ & $100 \sim 102$ & White solid & 61.0 & $71.23(70.91)$ & $7.25(7.44)$ & $13.64(13.78)$ \\
\hline
\end{tabular}

表 2 化合物 $\mathbf{2 a} \sim \mathbf{2 0}$ 的 ${ }^{1} \mathrm{H}$ NMR 和 IR 分析数据

Table $2{ }^{1} \mathrm{H}$ NMR and IR spectral data of compounds $\mathbf{2 a} \sim \mathbf{2 0}$

\begin{tabular}{|c|c|c|}
\hline Compd. & ${ }^{1} \mathrm{H}$ NMR $\delta$ & IR $v / \mathrm{cm}^{-1}$ \\
\hline $2 a$ & $\begin{array}{l}1.86 \sim 1.90(\mathrm{~m}, 2 \mathrm{H}, 6-\mathrm{H}), 2.35(\mathrm{brs}, 8 \mathrm{H}, \mathrm{pip}-\mathrm{H}), 2.62(\mathrm{t}, J=6.3 \mathrm{~Hz}, 2 \mathrm{H}, 5-\mathrm{H}), 3.12(\mathrm{~s}, 2 \mathrm{H}, \\
\left.\mathrm{NHCOCH}_{2}\right), 3.36(\mathrm{~s}, 2 \mathrm{H}, 8-\mathrm{H}), 3.79(\mathrm{t}, J=6.2 \mathrm{~Hz}, 2 \mathrm{H}, 7-\mathrm{H}), 7.08 \sim 7.21(\mathrm{~m}, 4 \mathrm{H}, \mathrm{ArH}), 7.30(\mathrm{t}, \\
J=8.1 \mathrm{~Hz}, 2 \mathrm{H}, \mathrm{ArH}), 7.42(\mathrm{brs}, 1 \mathrm{H}, 3-\mathrm{H}), 7.54(\mathrm{~d}, J=8.1 \mathrm{~Hz}, 2 \mathrm{H}, \mathrm{ArH}), 9.10(\mathrm{~s}, 1 \mathrm{H}, \mathrm{NH})\end{array}$ & $\begin{array}{l}3256,2936,2821, \\
1699,1650,1521,760\end{array}$ \\
\hline $2 b$ & $\begin{array}{l}1.99 \sim 2.01(\mathrm{~m}, 2 \mathrm{H}, 6-\mathrm{H}), 2.66(\mathrm{brs}, 8 \mathrm{H}, \mathrm{pip}-\mathrm{H}), 2.71(\mathrm{t}, J=6.3 \mathrm{~Hz}, 2 \mathrm{H}, 5-\mathrm{H}), 3.16(\mathrm{~s}, 2 \mathrm{H}, \\
\left.\mathrm{NHCOCH}_{2}\right), 3.38(\mathrm{~s}, 2 \mathrm{H}, 8-\mathrm{H}), 3.80(\mathrm{t}, J=6.2 \mathrm{~Hz}, 2 \mathrm{H}, 7-\mathrm{H}), 7.10 \sim 7.29(\mathrm{~m}, 4 \mathrm{H}, \mathrm{ArH}), 7.41 \text { (brs, } \\
1 \mathrm{H}, 3 \mathrm{H}), 7.94(\mathrm{t}, J=8.1 \mathrm{~Hz}, 1 \mathrm{H}, \operatorname{ArH}), 7.20(\mathrm{~d}, J=8.1 \mathrm{~Hz}, 1 \mathrm{H}, \mathrm{ArH}), 9.33(\mathrm{~s}, 1 \mathrm{H}, \mathrm{NH})\end{array}$ & $\begin{array}{l}3268,2942,2821, \\
1697,1649,1537,760\end{array}$ \\
\hline $2 c$ & $\begin{array}{l}1.47\left(\mathrm{~d}, J=7.5 \mathrm{~Hz}, 3 \mathrm{H}, \mathrm{CH}_{3}\right), 1.95 \sim 1.99(\mathrm{~m}, 2 \mathrm{H}, 6-\mathrm{H}), 2.52(\mathrm{brs}, 8 \mathrm{H}, \mathrm{pip}-\mathrm{H}), 2.71(\mathrm{t}, J=6.3 \mathrm{~Hz}, \\
2 \mathrm{H}, 5-\mathrm{H}), 2.98(\mathrm{~d}, J=6.2 \mathrm{~Hz}, 2 \mathrm{H}, \mathrm{NHCOCH}), 3.31(\mathrm{~s}, 2 \mathrm{H}, 8-\mathrm{H}), 3.78(\mathrm{t}, J=6.2 \mathrm{~Hz}, 2 \mathrm{H}, 7-\mathrm{H}), \\
5.12 \sim 5.17(\mathrm{~m}, 1 \mathrm{H}, \mathrm{CH}), 7.08 \sim 7.19(\mathrm{~m}, 3 \mathrm{H}, \mathrm{ArH}), 7.23 \sim 7.36(\mathrm{~m}, 6 \mathrm{H}, \mathrm{ArH}), 7.40(\mathrm{~d}, J=8.1 \mathrm{~Hz}, \\
1 \mathrm{H}, \mathrm{NH})\end{array}$ & $\begin{array}{l}3271,2939,2820, \\
1701,1650,1539,763\end{array}$ \\
\hline $2 d$ & $\begin{array}{l}1.98 \sim 2.00(\mathrm{~m}, 2 \mathrm{H}, 6-\mathrm{H}), 2.70(\mathrm{brs}, 8 \mathrm{H}, \mathrm{pip}-\mathrm{H}), 2.70(\mathrm{t}, J=6.3 \mathrm{~Hz}, 2 \mathrm{H}, 5-\mathrm{H}), 3.23(\mathrm{~s}, 2 \mathrm{H}, \\
\left.\mathrm{NHCOCH}_{2}\right), 3.37(\mathrm{~s}, 2 \mathrm{H}, 8-\mathrm{H}), 3.79(\mathrm{t}, J=6.2 \mathrm{~Hz}, 2 \mathrm{H}, 7-\mathrm{H}), 7.13 \sim 7.22(\mathrm{~m}, 3 \mathrm{H}, \mathrm{ArH}), 7.26(\mathrm{brs}, \\
1 \mathrm{H}, 3 \mathrm{H}), 7.85(\mathrm{~d}, J=8.7 \mathrm{~Hz}, 1 \mathrm{H}, \mathrm{ArH}), 8.52(\mathrm{~s}, 1 \mathrm{H}, \mathrm{ArH}), 9.08(\mathrm{~d}, J=9.0 \mathrm{~Hz}, 1 \mathrm{H}, \mathrm{ArH}), 12.10(\mathrm{~s}, \\
1 \mathrm{H}, \mathrm{NH})\end{array}$ & $\begin{array}{l}3269,2938,2821, \\
1711,1656,1516,750\end{array}$ \\
\hline $2 e$ & $\begin{array}{l}1.99 \sim 2.01(\mathrm{~m}, 2 \mathrm{H}, 6-\mathrm{H}), 2.69(\mathrm{brs}, 8 \mathrm{H}, \mathrm{pip}-\mathrm{H}), 2.70(\mathrm{t}, J=6.3 \mathrm{~Hz}, 2 \mathrm{H}, 5-\mathrm{H}), 3.21(\mathrm{~s}, 2 \mathrm{H}, \\
\mathrm{NHCOCH} \\
), 3.38(\mathrm{~s}, 2 \mathrm{H}, 8-\mathrm{H}), 3.79(\mathrm{t}, J=6.2 \mathrm{~Hz}, 2 \mathrm{H}, 7-\mathrm{H}), 7.16 \sim 7.19(\mathrm{~m}, 4 \mathrm{H}, \mathrm{ArH}), 7.26(\mathrm{brs}, \\
1 \mathrm{H}, 3 \mathrm{H}), 7.68(\mathrm{t}, J=0.9 \mathrm{~Hz}, 1 \mathrm{H}, \operatorname{ArH}), 8.21(\mathrm{~d}, J=8.4 \mathrm{~Hz}, 1 \mathrm{H}, \operatorname{ArH}), 8.85(\mathrm{~d}, J=8.7 \mathrm{~Hz}, 1 \mathrm{H}, \\
\text { ArH), } 11.86(\mathrm{~s}, 1 \mathrm{H}, \mathrm{NH})\end{array}$ & $\begin{array}{l}3240,2939,2823, \\
1698,1656,1500,745\end{array}$ \\
\hline
\end{tabular}




\begin{tabular}{cl}
\hline Compd. & \multicolumn{1}{c}{${ }^{1} \mathrm{H}$ NMR $\delta$} \\
\hline \multirow{2}{*}{$\mathbf{2 f} \quad$} & $1.98 \sim 2.00(\mathrm{~m}, 2 \mathrm{H}, 6-\mathrm{H}), 2.21\left(\mathrm{~s}, 6 \mathrm{H}, \mathrm{CH}_{3}\right), 2.68(\mathrm{brs}, 8 \mathrm{H}, \mathrm{pip}-\mathrm{H}), 2.71(\mathrm{t}, J=6.3 \mathrm{~Hz}, 2 \mathrm{H}, 5-\mathrm{H})$, \\
& $3.19(\mathrm{~s}, 2 \mathrm{H}, 8-\mathrm{H}), 3.35(\mathrm{~s}, 2 \mathrm{H}, 8-\mathrm{H}), 3.79(\mathrm{t}, J=6.2 \mathrm{~Hz}, 2 \mathrm{H}, 7-\mathrm{H}), 7.08 \sim 7.18(\mathrm{~m}, 6 \mathrm{H}, \mathrm{ArH}), 7.26$ \\
& $(\mathrm{brs}, 1 \mathrm{H}, 3 \mathrm{H}), 8.64(\mathrm{~s}, 1 \mathrm{H}, \mathrm{NH})$ \\
& $1.98 \sim 2.00(\mathrm{~m}, 2 \mathrm{H}, 6-\mathrm{H}), 2.66(\mathrm{brs}, 8 \mathrm{H}, \mathrm{pip}-\mathrm{H}), 2.70(\mathrm{t}, J=6.3 \mathrm{~Hz}, 2 \mathrm{H}, 5-\mathrm{H}), 3.19(\mathrm{~s}, 2 \mathrm{H}$, \\
$\mathbf{2 g} \quad$ & $\mathrm{NHCOCH}), 3.35(\mathrm{~s}, 2 \mathrm{H}, 8-\mathrm{H}), 3.79(\mathrm{t}, J=6.2 \mathrm{~Hz}, 2 \mathrm{H}, 7-\mathrm{H}), 3.95\left(\mathrm{~s}, 6 \mathrm{H}, \mathrm{CH}_{3}\right), 5.80(\mathrm{~s}, 1 \mathrm{H}, \mathrm{Py}-\mathrm{H})$, \\
& $7.12 \sim 7.19(\mathrm{~m}, 3 \mathrm{H}, \mathrm{ArH}), 7.26(\mathrm{brs}, 1 \mathrm{H}, 3 \mathrm{H}), 9.39(\mathrm{~s}, 1 \mathrm{H}, \mathrm{NH})$ \\
& $1.97 \sim 2.01(\mathrm{~m}, 2 \mathrm{H}, 6-\mathrm{H}), 2.68(\mathrm{brs}, 8 \mathrm{H}, \mathrm{pip}-\mathrm{H}), 2.72(\mathrm{t}, J=6.3 \mathrm{~Hz}, 2 \mathrm{H}, 5-\mathrm{H}), 3.21(\mathrm{~s}, 2 \mathrm{H}$, \\
& $\mathrm{NHCOCH}), 3.34(\mathrm{~s}, 2 \mathrm{H}, 8-\mathrm{H}), 3.80(\mathrm{t}, J=6.2 \mathrm{~Hz}, 2 \mathrm{H}, 7-\mathrm{H}), 7.13 \sim 7.22(\mathrm{~m}, 3 \mathrm{H}, \mathrm{ArH}), 7.27(\mathrm{brs}$, \\
$\mathbf{2 h} \quad$ & $1 \mathrm{H}, 3 \mathrm{H}), 8.43(\mathrm{~d}, J=9.0 \mathrm{~Hz}, 1 \mathrm{H}, \mathrm{ArH}), 8.52(\mathrm{~d}, J=2.7 \mathrm{~Hz}, 1 \mathrm{H}, \mathrm{ArH}), 8.83(\mathrm{~d}, J=9.0 \mathrm{~Hz}, 1 \mathrm{H}$, \\
& $\mathrm{ArH}), 10.40(\mathrm{~s}, 1 \mathrm{H}, \mathrm{NH})$ \\
& $1.91 \sim 1.96(\mathrm{~m}, 2 \mathrm{H}, 6-\mathrm{H}), 2.52(\mathrm{brs}, 8 \mathrm{H}, \mathrm{pip}-\mathrm{H}), 2.67(\mathrm{t}, J=6.3 \mathrm{~Hz}, 2 \mathrm{H}, 5-\mathrm{H}), 3.06(\mathrm{~s}, 2 \mathrm{H}$, \\
$\mathbf{2} \mathbf{2 i} \quad$ & $\mathrm{NHCOCH}), 3.24(\mathrm{~s}, 2 \mathrm{H}, 8-\mathrm{H}), 3.75(\mathrm{t}, J=6.2 \mathrm{~Hz}, 2 \mathrm{H}, 7-\mathrm{H}), 7.08 \sim 7.14(\mathrm{~m}, 4 \mathrm{H}, \mathrm{ArH}), 7.16 \sim 7.34$ \\
& $(\mathrm{~m}, 10 \mathrm{H}, \mathrm{ArH})$ \\
& $1.87 \sim 1.93(\mathrm{~m}, 2 \mathrm{H}, 6-\mathrm{H}), 2.50(\mathrm{brs}, 8 \mathrm{H}, \mathrm{pip}-\mathrm{H}), 2.70(\mathrm{t}, J=6.3 \mathrm{~Hz}, 2 \mathrm{H}, 5-\mathrm{H}), 3.12(\mathrm{~s}, 2 \mathrm{H}$, \\
$\mathbf{2} \mathbf{2 j} \quad \mathrm{NHCOCH}), 3.35(\mathrm{~s}, 2 \mathrm{H}, 8-\mathrm{H}), 3.69(\mathrm{t}, J=6.2 \mathrm{~Hz}, 2 \mathrm{H}, 7-\mathrm{H}), 7.12 \sim 7.18(\mathrm{~m}, 5 \mathrm{H}, \mathrm{ArH}), 7.36 \sim 7.40$ \\
& $(\mathrm{~m}, 1 \mathrm{H}, \mathrm{ArH}), 7.58(\mathrm{brs}, 1 \mathrm{H}, 3 \mathrm{H}), 9.42(\mathrm{~s}, 1 \mathrm{H}, \mathrm{NH})$
\end{tabular}

$1.97 \sim 1.99(\mathrm{~m}, 2 \mathrm{H}, 6-\mathrm{H}), 2.64$ (brs, 8H, pip-H), 2.71 (t, $J=6.3 \mathrm{~Hz}, 2 \mathrm{H}, 5-\mathrm{H}), 3.11$ (s, 2H, $\left.\mathrm{NHCOCH}_{2}\right), 3.35(\mathrm{~s}, 2 \mathrm{H}, 8-\mathrm{H}), 3.79\left(\mathrm{~s}, 3 \mathrm{H}, \mathrm{CH}_{3}\right), 3.79(\mathrm{t}, J=6.2 \mathrm{~Hz}, 2 \mathrm{H}, 7-\mathrm{H}), 6.85 \sim 6.88(\mathrm{~m}$, $2 \mathrm{H}, \mathrm{ArH}), 7.16 \sim 7.22(\mathrm{~m}, 3 \mathrm{H}, \mathrm{ArH}), 7.45(\mathrm{~d}, J=9.0 \mathrm{~Hz}, 2 \mathrm{H}, \mathrm{ArH}), 8.97$ (s, 1H, NH)

$1.98 \sim 2.00(\mathrm{~m}, 2 \mathrm{H}, 6-\mathrm{H}), 2.31\left(\mathrm{~s}, 3 \mathrm{H}, \mathrm{CH}_{3}\right), 2.64$ (brs, 8H, pip-H), 2.7 (t, $\left.J=6.3 \mathrm{~Hz}, 2 \mathrm{H}, 5-\mathrm{H}\right)$,

2l $\quad 3.11\left(\mathrm{~s}, 2 \mathrm{H}, \mathrm{NHCOCH}_{2}\right), 3.35(\mathrm{~s}, 2 \mathrm{H}, 8-\mathrm{H}), 3.79\left(\mathrm{~s}, 3 \mathrm{H}, \mathrm{CH}_{3}\right), 3.79(\mathrm{t}, J=6.2 \mathrm{~Hz}, 2 \mathrm{H}, 7-\mathrm{H}), 7.11$ $7.16(\mathrm{~m}, 5 \mathrm{H}, \mathrm{ArH}), 7.42$ (d, $J=8.1 \mathrm{~Hz}, 2 \mathrm{H}, \mathrm{ArH}), 9.02(\mathrm{~s}, 1 \mathrm{H}, \mathrm{NH})$

$1.96 \sim 2.00(\mathrm{~m}, 2 \mathrm{H}, 6-\mathrm{H}), 2.67$ (brs, 8H, pip-H), 2.73 (t, $J=6.3 \mathrm{~Hz}, 2 \mathrm{H}, 5-\mathrm{H}), 3.18(\mathrm{~s}, 2 \mathrm{H}$,

$2 \mathbf{m}$ $\left.\mathrm{NHCOCH}_{2}\right), 3.39(\mathrm{~s}, 2 \mathrm{H}, 8-\mathrm{H}), 3.79(\mathrm{t}, J=6.2 \mathrm{~Hz}, 2 \mathrm{H}, 7-\mathrm{H}), 7.13 \sim 7.22(\mathrm{~m}, 3 \mathrm{H}$, ArH), 7.26 (brs, $1 \mathrm{H}, 3 \mathrm{H}), 7.96 \sim 8.02(\mathrm{~m}, 3 \mathrm{H}, \mathrm{ArH}), 9.58(\mathrm{~s}, 1 \mathrm{H}, \mathrm{NH})$

$1.98 \sim 2.02(\mathrm{~m}, 2 \mathrm{H}, 6-\mathrm{H}), 2.67$ (brs, 8H, pip-H), 2.71 (t, $J=6.3 \mathrm{~Hz}, 2 \mathrm{H}, 5-\mathrm{H}), 3.16$ (s, 2H, $\left.\mathrm{NHCOCH}_{2}\right), 3.38(\mathrm{~s}, 2 \mathrm{H}, 8-\mathrm{H}), 3.80(\mathrm{t}, J=6.2 \mathrm{~Hz}, 2 \mathrm{H}, 7-\mathrm{H}), 7.13 \sim 7.22(\mathrm{~m}, 3 \mathrm{H}, \mathrm{ArH}), 7.26$ (brs,

2n $1 \mathrm{H}, 3 \mathrm{H}), 7.48 \sim 7.52(\mathrm{~m}, 1 \mathrm{H}, \mathrm{ArH}), 7.96(\mathrm{~d}, J=8.4 \mathrm{~Hz}, 1 \mathrm{H}, \mathrm{ArH}), 8.01(\mathrm{~d}, J=8.4 \mathrm{~Hz}, 1 \mathrm{H}, \mathrm{ArH})$, $8.3(\mathrm{~d}, J=1.8 \mathrm{~Hz}, 1 \mathrm{H}, \mathrm{ArH}), 9.38(\mathrm{~s}, 1 \mathrm{H}, \mathrm{NH})$

$1.93 \sim 1.97$ (m, 2H, 6-H), 2.52 (brs, 8H, pip-H), 2.68 (t, J=6.3 Hz, 2H, 5-H), 2.91 (s, 2H,

$\left.20 \mathrm{NHCOCH}_{2}\right), 3.25\left(\mathrm{~s}, 5 \mathrm{H}, 8-\mathrm{H}_{,} \mathrm{CH}_{3}\right), 3.76(\mathrm{t}, J=6.3 \mathrm{~Hz}, 2 \mathrm{H}, 7-\mathrm{H}), 7.09 \sim 7.20(\mathrm{~m}, 5 \mathrm{H}, \mathrm{ArH})$, $7.33 \sim 7.43(\mathrm{~m}, 4 \mathrm{H}, \mathrm{ArH})$
$\operatorname{IR} v / \mathrm{cm}^{-1}$

$3241,2939,2819$,

$1668,1654,1492,764$

$3312,2937,2822$, $1676,1654,1514,763$

$3306,2942,2826$, $1716,1654,1511,749$

2926, 2810, 16671491 , 750

3276, 2940, 2821, $1713,1654,1513$, 1011,763

$3300,2937,2822$, $1715,1654,1514$ 1245,763

$3301,2937,2820$, $1708,1655,1521,750$

$3298,2936,2822$, 1709, 1657, 1521, $1324,1275,750$

$3302,2938,2821$ $1702,1649,1529,750$

2921, 2810, 1655, $1494,1275,1260,750$

\section{3 目标化合物 $2 \mathrm{a} \sim 20$ 的生物活性测定}

1.3.1 对小菜蛾(Plutella xylostella) 的生物活性测定 (浸叶法)

采用国际抗性行动委员会(IRAC)提出的浸叶法. 用 直头眼科镊子夹取甘蓝叶片浸渍到配制好的待测药液 中, 时间 3 5 s, 甩掉余液. 每次 1 片, 每个样品共 3 片. 按样品标记顺序依次放在处理纸上. 待药液干后, 放入 具有标记的 $10 \mathrm{~cm}$ 长的直型管内, 接入 2 龄小菜蛾幼虫 30 头, 用纱布盖好管口. 将试验处理置于标准处理室 内, $96 \mathrm{~h}$ 检查结果以拨针轻触虫体, 不动者为死亡. 计 算死亡率，实验做 3 次重复，取平均值. 采用空白对照, 并采用商品化杀虫剂噻虫嗪和吡虫啉作为对照药剂.
1.3.2 对甜菜夜蛾 (Spodoptera exigua) 的生物活性测 定(浸叶法)

采用国际抗性行动委员会(IRAC)提出的浸叶法. 用 直头眼科铌子夹取甘蓝叶片浸渍到配制好的待测药液 中，时间 3 5 s, 甩掉余液. 每次 1 片，每个处理共 3 片. 按样品标记顺序依次放在处理纸上. 待药液干后, 放入 具有标记的 $60 \mathrm{~cm}$ 长的培养血内, 接入 3 龄甜菜夜蛾幼 虫 30 头, 盖好盖子. 将试验处理置于标准处理室内, 96 $\mathrm{h}$ 检查结果以拨针轻触虫体，不动者为死亡. 计算死亡 率，实验做 3 次重复，取平均值. 采用空白对照，并采用 商品化杀虫剂噻虫嗪和吡虫啉作为对照药剂. 
1.3.3 对粘虫 (Mythimna separate) 的生物活性测定 (浸叶法)

将样品用丙酮配制成不同浓度的溶液, 浸渍苗期玉 米叶, 晾干后放入 $7 \mathrm{~cm}$ 培养血中, 接入 4 龄幼虫, 重复 2 4 次. 对照用丙酮溶液浸渍玉米叶饲养幼虫. $24 \mathrm{~h}$ 后 随时添加新鲜的玉米叶片. 在 $24,48,72,96 \mathrm{~h}$ 观察试验 结果, 直至对照幼虫正常蜕皮变成 5 龄. 以粘虫幼虫完全 死亡, 即触之不动为幼虫的死亡标准. 计算死亡率, 实 验做 3 次重复, 取平均值. 采用空白对照, 并采用商品 化杀虫剂噻虫嗪和吡虫啉作为对照药剂.

1.3.4 对玉米螟 (Pyrausta nubilalis) 的生物活性测定 (浸虫法)

将样品用丙酩配制成不同浓度的溶液, 将适龄试虫 放于浸虫器内于药液中浸渍 3 $5 \mathrm{~s}$, 放入加有人工饲料 的十孔养虫盒内放于恒温养虫室中饲养. 处理后 $72 \mathrm{~h}$ 检 查死、活虫数, 计算死亡率, 实验做 3 次重复, 取平均值. 采用空白对照, 并采用商品化杀虫剂噻虫嗪和吡虫啉作 为对照药剂.

1.3.5 对棉铃虫 (Helicoverpa armigera) 生物活性测定 (微量点滴法)

采用 FAO 推荐微量点滴法, 用丙酮将原药稀释成 4 5 个系列浓度, 选取发育一致的试虫用 Robbins Scientific 微量点滴器将药液点滴到幼虫的胸部背板, 3 龄幼虫每头 $0.5 \mu \mathrm{L}, 4$ 龄幼虫每头 $1.0 \mu \mathrm{L}$, 处理后 $72 \mathrm{~h}$ 检 查死、活虫数, 计算死亡率, 实验做 3 次重复, 取平均值. 采用空白对照, 并采用商品化杀虫剂噻虫嗪和吡虫啉作 为对照药剂.

1.3.6 对蚊幼虫(Culex pipiens pallens Coquillett)的生 物活性测定

将样品用丙酮配制成一定浓度的母液, 在 $100 \mathrm{~mL}$ 的烧杯内加入 $90 \mathrm{~mL}$ 脱氯水(自来水静置 $15 \mathrm{~d}$ 以上), 分 别加入不同量的样品母液, 再加 $10 \mathrm{~mL}$ 含有 10 头 4 龄 蚊幼虫 (2 日龄, 虫体腹部变粗)的饲养水. 处理后每天添 加少许饲料, 直到蚊幼虫全部化蛹或死亡. 处理室温度 $(28 \pm 2){ }^{\circ} \mathrm{C}$. 随时将化的蛹吸走, 观察时间为 $8 \mathrm{~d}$, 计算 死亡率, 实验做 3 次重复, 取平均值. 采用空白对照, 并 采用商品化杀虫剂噻虫嗪和吡虫啉作为对照药剂.

\subsection{7 目标化合物的杀菌活性测定}

采用离体平皿法 ${ }^{[5]}$, 以四种常见植物病原菌水稻纹 枯菌(Thanatephorus cucumeris)、黄瓜灰霉菌(Botrytis cinerea)、小麦赤雱菌(Fusarium graminearum)、番茄早 疫病(Tomato Early Blight)、烟草赤星病[Alternaria alternata (Fries) Keissler]、黄瓜炭疽病菌(Colletotrichum acutatum) 为对象, 对所合成的化合物进行杀菌活性测 定. 药液以 $N, N$-二甲基甲酰胺为溶剂, 实验终浓度为 50 $\mu \mathrm{g} / \mathrm{mL}$. 将配制好的药液各取 $1 \mathrm{~mL}$ 于已灭菌的培养血 中, 再取冷却到 $45{ }^{\circ} \mathrm{C}$ 左右的 PDA 培养基 $9 \mathrm{~mL}$ 倒入培 养血, 充分混匀, 置平板使凝固. 然后用打孔器移接菌 饼，菌面向下接于含毒培养基中心，每具一块，放于恒 温箱中，培养 $2 \mathrm{~d}$ 后测量菌落直径，通过以下公式计算 抑制率.

抑制率 $\%=($ 对照菌落直径一处理菌落直径 $) \div$ 对照 菌落直径 $\times 100 \%$

每个样品重复 3 次，以加入无菌水的培养基为对照， 分别采用 $70 \%$ 甲基托布津 WP, 40\%菌核净 WP, 75\%百 菌清 WP 和 $50 \%$ 多菌灵 WP 为对照药剂.

\section{2 结果与讨论}

\subsection{Aspernigerin 类似物的合成}

\subsection{1 目标化合物 2 合成路线的选择}

针对目标化合物 2 的结构特点, 不难发现其结构是 一类以哌溙环为中心的对称性化合物. 因此, 在目标化 合物的合成路线设计上，我们有两种选择：一是参考谭 仁祥课题组合成 Aspernigerin 的方法 ${ }^{[1,2]}$, 以哌嗪为原料, 由中心逐步向外合成的方法(Scheme 2), 该方法路线较 长, 操作较为繁琐, 收率较低, 仅为 $47.3 \%$, 产物纯化困 难，尤其是在合成中间体 I 和 II 时，因其氨基酰氯结构， 很不稳定, 且是以盐酸盐的形式存在, 难以进行纯化, 只能一步合成到目标产物 $\mathbf{2}$ 后再来进行纯化. 二是根据 我们前期工作中完成的 Aspernigerin 全合成新方法, 采 用由外及里的合成方法, 先合成氯乙酰基-1,2,3,4-四氢 喹啉和氯乙酰基芳胺片段，再与哌溙中心对接，不但步 骤简便，而且每步中间体都易于纯化(Scheme 1). 经过 两种方法的探索, 最后, 我们选定 Scheme 1 所示路线作 为目标化合物 $\mathbf{2}$ 的最终合成路线.

2.1.2中间体 $N$-氯乙酰-1,2,3,4-四氢咺啉的合成

参照文献[6,7]方法合成中间体 $N$-氯乙酰-1,2,3,4-四 氢喹啉时，中间体是不经过纯化直接用于下一步合成 的. 但是, 当我们用不经过纯化的 $N$-氯乙酰-1,2,3,4-四 氢喹啉直接用于后一步反应时, 发现产物很复杂, 而且 不好纯化, 需要通过多次柱层析纯化才能得到纯度较好 的产品, 收率很低, 而用纯化好的 $N$-氯乙酰-1,2,3,4-四 氢喹啉作原料后, 反应副产物大大减少. 为此, 我们针 对中间体 $N$-氯乙酰-1,2,3,4-四氢喹啉的纯化进行了细致 的研究, 首先, 我们采用柱层析的方法进行纯化, 可以 获得较高纯度的 $N$-氯乙酰-1,2,3,4-四氢哇啉, 但是收率 较低, 一次得到的中间体量较少. 而后, 我们发现纯的 $N$-氯乙酰-1,2,3,4-四氢喹啉在常温下是液体, 尝试性地 进行高真空减压蒸馏, 收集 $162 \sim 164{ }^{\circ} \mathrm{C} / 130 \mathrm{~Pa}$ 的主要 馏分, 经结构鉴定即为产物, 不但纯度高, 而且收率较 
<smiles>CC(C)C(=O)CN1CCN(CC(=O)O[Na])CC1</smiles><smiles>O=C(O)CN1CCN(CC(=O)O)CC1</smiles><smiles>O=C(Cl)CN1CCN(CC(=O)Cl)CC1</smiles><smiles>c1ccc2c(c1)CCCN2</smiles><smiles>O=C(Cl)CN1CCN(CC(=O)N2CCCc3ccccc32)CC1</smiles>

ArNHR<smiles>[R]N([Al])C(=O)CN1CCN(CC(=O)N2CCCc3ccccc32)CC1</smiles>

I

Scheme 2

柱层析也有较大提高, 操作简便, 能一次性得到大量的 $N$-氯乙酰-1,2,3,4-四氢喹啉.

\subsection{3 $N$ - $(1,2,3,4$-四氢喹啉基- $N$-甲酰甲基)哌嗪的合 成}

在合成 $N$-(1,2,3,4-四氢喹啉基- $N$-甲酰甲基)哌嗪的 反应过程中, 容易发生产物和 $N$-氯乙酰-1,2,3,4-四氢喹 啉进一步反应生成 Aspernigerin 的副反应, 而原料哌溙 的过量可抑制该副反应的发生, 过量越多, 副产物就越 少, 但是到了一定程度后, 增加哌嗪的量对抑制副产物 的作用就越来越小, 且原料哌嗪的回收困难, 损失也大 大增加. 实验证明, 在摩尔投料比上哌溙为 $N$-氯乙酰1,2,3,4-四氢喹啉的 2 倍左右为宜.

反应溶剂的选择，该反应原理上是氮上的亲核取代 反应，根据文献[8～10]报道的常用溶剂，我们重点针对 无水乙醇、 $N, N$-二甲基甲酰胺和甲苯做了篮选实验，以 点板监控 $N$-氯乙酰-1,2,3,4-四氢喹啉消失为反应终点, 结果如表 3 所示.

\section{表 3 反应溶剂对收率的影响}

Table 3 Influence of reaction solvent on the yield

\begin{tabular}{lccc}
\hline \multicolumn{1}{c}{ 反应溶剂 } & $T /{ }^{\circ} \mathrm{C}$ & $t / \mathrm{h}$ & 产物收率\% \\
\hline 无水乙醇 & 80 & 4.0 & 46.6 \\
$N, N$ 二甲甲基甲酰胺 & 80 & 4.0 & 72.0 \\
甲苯 & 80 & 6.0 & 36.2 \\
\hline
\end{tabular}

从表 3 可以看出, 以非极性溶剂甲苯作溶剂的不但 反应时间长, 而且收率很低, 主要是因为反应原料哌嗪 在甲苯中的溶解度较差, 生成的产物容易 $N$-氯乙酰1,2,3,4-四氢喹啉进一步反应生成副产物 Aspernigerin; 以无水乙醇作溶剂的反应收率要比 $N, N$-二甲基甲酰胺 的反应收率低, 主要是因为质子化的溶剂存在参与竞争 反应的烷氧基负离子, 消耗了一部分原料, 导致收率下 降，最终确定该步反应溶剂为 $N, N$-二甲基甲酰胺.

\subsection{4 目标化合物的合成}

在目标化合物的合成中, 为了便于目标化合物的分 离纯化, 必须确保 $N$-(1,2,3,4-四氢喹啉基- $N$-甲酰甲基)
哌嗪反应完全，因此，在投料比上，以原料氯乙酰芳胺 过量为宜. 为了反应快速, 便于后处理, 在缚酸剂的选 择上, 我们选择了液体有机碱三乙胺作为缚酸剂, 效果 非常理想. 溶剂的选择上我们尝试过甲苯、三氯甲烷和 $N, N$-二甲基甲酰胺 ${ }^{[11,12]}$, 甲苯因为对 $N-(1,2,3,4$-四氢喹 啉基- $N$-甲酰甲基)哌嗪的溶解性较差，反应时间长，副 产物复杂, 分离纯化困难, 收率低; $N, N$-二甲基甲酰胺 作溶剂主要存在 $N, N$-二甲基甲酰胺沸点高，难以脱除， 且容易在产品中残留等问题; 最终我们选择了三氯甲烷 作溶剂进行反应，不但收率高，而且后处理简单，容易 得到高纯度目标化合物.

\section{2 目标化合物的图谱解析}

以目标化合物 $2 \mathbf{a}$ 为例, 在化合物 $\mathbf{2 a}$ 的红外图谱中, 在 $3256 \mathrm{~cm}^{-1}$ 处出现了 $\mathrm{N}-\mathrm{H}$ 伸缩振动吸收峰, 化合物 中亚甲基 $\mathrm{CH}_{2}$ 上的碳氢伸缩振动吸收峰在 2936, 2821 $\mathrm{cm}^{-1}$ 两处, $1699,1651 \mathrm{~cm}^{-1}$ 处为典型的 $\mathrm{C}=\mathrm{O}$ 伸缩振动 吸收, 而在 $1491 \mathrm{~cm}^{-1}$ 处出现的峰为 $\mathrm{CH}_{2}$ 上的碳氢弯曲 振动吸收峰, $\mathrm{C}-\mathrm{N}$ 伸缩振动吸收峰在 $1298 \mathrm{~cm}^{-1}$ 处.

在化合物 2a 的 ${ }^{1} \mathrm{H}$ NMR 中, 为了便于解析，我们对 化合物中的氢原子作如 Scheme 3 编号所示, 化合物中 唯一的活泼氢酰胺质子的化学位移值在 $\delta 9.1$ 处显示为 单峰, $\delta 7.5$ 处的两重峰和 $\delta 7.3$ 处的三重峰为苯胺芳环上 氢的化学位移值, 化合物中 3 位氢的化学位移值在 $\delta 7.4$ 左右, 图谱上显示为较宽的单峰, 我们分析认为是 3 位 氢受分子内酰胺羰基的影响, 形成分子内六元环结构, 由于氧原子的电负性强，产生吸电子效应，导致苯环上 3 位的 $\mathrm{C}-\mathrm{H}$ 键电子云密度降低, 不但化学位移向低场 移动, 而且具有了一定的活泼氢的性质, 峰型变宽. 芳 环上其他的质子在化学位移 $\delta 7.0 \sim 7.2$ 处显示为多重峰. 7 位质子受酰胺 $\mathrm{N}$ 原子的影响化学位移向低场移动, 并 受 6 位质子的偶合裂分，在化学位移 $\delta 3.7$ 左右显示为三 重峰. 8 位质子受酰胺羰基的影响化学位移向低场移动, 在化学位移 $\delta 3.4$ 处显示为单峰, 而化合物中另一个乙 酰基的 $\mathrm{CH}_{2}$ 质子在化学位移 $\delta 3.1$ 处同样显示为单峰. 5 
位质子受苯环的影响化学位移也在相对低场为 $\delta 2.6$ 左 右. 哌嗪环上的质子在化学位移 $\delta 2.3$ 处显示为较宽的 单峰. 6 位质子化学位移在最高场为 $\delta 1.9$ 左右, 由于受 5,7 位多个质子的偶合裂分，显示为多重峰.

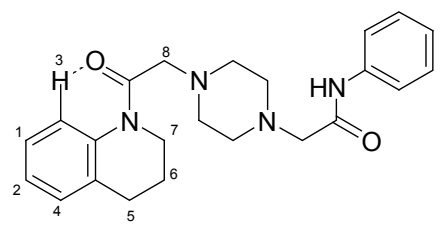

Scheme 3

\section{3 目标化合物的生物活性}

\subsection{1 目标化合物的杀虫活性}

分别采用浸叶法、浸渍法、浸虫法、微量点滴法测 定了目标化合物 $\mathbf{2 a} \sim \mathbf{2 0}$ 在 $600 \mu \mathrm{g} / \mathrm{mL}$ 的浓度下对小菜 蛾、甜菜夜蛾、蚜虫、红蜘蛛、粘虫、棉铃虫、玉米螟、 蚊幼虫的生物活性, 并采用商品化杀虫剂噻虫嗪和吡虫 啉作为对照药剂, 其测定结果列于表 4 中.

由表 4 中结果可知：大部分目标化合物在 600 $\mu \mathrm{g} / \mathrm{mL}$ 的浓度下对小菜蛾、甜菜夜蛾、粘虫、棉铃虫、 玉米蛽和蚊幼虫表现出了一定的杀虫活性, 其中化合物 $2 n$ 对小菜蛾的致死率在 $600,200 \mu \mathrm{g} / \mathrm{mL}$ 的浓度下对小 菜蛾表现出 $100 \%$ 的杀虫活性, 高于母体化合物 Aspernigerin 和对照药剂噻虫嗪. 值得关注的是, 杀虫 活性普遍表明 $\mathbf{2 0} \geqslant \mathbf{2} i \geqslant \mathbf{2} a$, 说明 $\mathrm{R}$ 基团对杀虫活性影响 是较为明显的, 具有 $\mathrm{CH}_{3} \geqslant \mathrm{C}_{6} \mathrm{H}_{5} \geqslant \mathrm{H}$ 的趋势, 因此, 在
以后的化合物设计中，可考虑 $\mathrm{R}$ 基团以不同的烷基取 代.

\subsection{2 目标化合物的杀菌活性}

采用离体平皿法 ${ }^{[5]}$, 测定了目标化合物 $\mathbf{2 a} \sim \mathbf{2 0}$ 在 $100 \mu \mathrm{g} / \mathrm{mL}$ 的浓度下对植物病原菌水稻纹枯菌 (Thanatephorus cucumeris)、黄瓜灰霉菌 (Botrytis cinerea)、小麦赤霉菌(Fusarium graminearum)、番茄早 疫病 (Tomato Early Blight)、烟草赤星病 (Alternaria alternata (Fries) Keissler)、黄瓜炭疽病菌(Colletotrichum acutatum) 的生物活性. 并采用商品化杀菌剂苯醚甲环唑 作为对照药剂, 其测定结果列于表 5 中.

由表 5 结果可知: 化合物 $\mathbf{2 d} \sim \mathbf{2 k}$ 在 $100 \mu \mathrm{g} / \mathrm{mL}$ 的 浓度下对植物病原菌小麦赤霉病、水稻纹枯菌、黄瓜灰 霉病、番茄早疫病、烟草赤星病、黄瓜炭疽病菌都表现 出了一定的抑制活性, 所有化合物都对黄瓜灰霉病表现 出了抑制活性, 其中化合物 $\mathbf{2 d}, \mathbf{2 e}, \mathbf{2 j}, \mathbf{2 k}$ 更是表现出了 中等偏上的抑制活性，抑制率分别为 $75 \%, 67 \%, 75 \%$, $62 \%$ ，优于母体化合物 Aspernigerin 的抑制活性，但是都 比对照药剂苯醚甲环唑的抑制活性差. 同样值得关注的 是，杀菌活性普遍表明 $\mathbf{2} \mathbf{i} \geqslant \mathbf{2 0} \geqslant \mathbf{2} a$, 说明 $\mathrm{R}$ 基团对杀菌 活性也有明显的影响, 具有 $\mathrm{C}_{6} \mathrm{H}_{5} \geqslant \mathrm{CH}_{3} \geqslant \mathrm{H}$ 的趋势, $\mathrm{R}$ 基 才越大活性越高，因此，在以后的化合物设计中，可考 虑 $\mathrm{R}$ 基团以较大基团取代.

以上研究结果表明, Aspernigerin 结构简单, 在医药 上表现出对肿瘤细胞有较好的抑制活性 ${ }^{[1,2]}$, 但在农业 上的应用研究未有报道. 我们以 Aspernigerin 为母体结

表 4 Aspernigerin 的类似物杀虫活性(\%)测试结果

Table 4 Insecticidal activity (\%) of aspernigerin's analogues

\begin{tabular}{|c|c|c|c|c|c|c|c|}
\hline \multirow{2}{*}{ Compd. } & \multicolumn{2}{|c|}{ 小菜蛾 } & \multirow{2}{*}{ 甜菜夜蛾 ${ }^{a}$} & \multirow{2}{*}{ 粘虫 ${ }^{a}$} & \multirow{2}{*}{ 棉铃虫 ${ }^{a}$} & \multirow{2}{*}{ 玉米蚯 ${ }^{a}$} & \multirow{2}{*}{ 蚊幼虫 ${ }^{b}$} \\
\hline & $600 \mu \mathrm{g} / \mathrm{mL}$ & $200 \mu \mathrm{g} / \mathrm{mL}$ & & & & & \\
\hline Aspernigerin & 100 & 75 & 21 & 20 & 20 & 40 & 50 \\
\hline $2 \mathbf{a}$ & 0 & 0 & 16 & 30 & 20 & 40 & 20 \\
\hline $2 b$ & 0 & 0 & 19 & 40 & 50 & 30 & 20 \\
\hline $2 c$ & 0 & 0 & 24 & 10 & 30 & 10 & 20 \\
\hline 2d & 0 & 0 & 31 & 20 & 20 & 40 & 40 \\
\hline $2 \mathrm{e}$ & 0 & 0 & 29 & 30 & 40 & 20 & 20 \\
\hline $2 f$ & 52 & 29 & 20 & 20 & 30 & 30 & 10 \\
\hline $2 \mathrm{~g}$ & 0 & 0 & 17 & 30 & 20 & 60 & 20 \\
\hline $2 \mathrm{~h}$ & 0 & 0 & 25 & 40 & 20 & 50 & 40 \\
\hline $2 \mathbf{i}$ & 36 & 14 & 21 & 20 & 30 & 30 & 50 \\
\hline $2 j$ & 42 & 14 & 29 & 10 & 40 & 20 & 10 \\
\hline $2 k$ & 38 & 14 & 27 & 20 & 50 & 20 & 30 \\
\hline 21 & 76 & 57 & 22 & 20 & 50 & 30 & 40 \\
\hline $2 m$ & 0 & 0 & 28 & 50 & 50 & 20 & 60 \\
\hline $2 n$ & 100 & 100 & 26 & 10 & 20 & 30 & 30 \\
\hline 20 & 58 & 43 & 24 & 20 & 20 & 30 & 50 \\
\hline 98\%噻虫嗪 & 100 & 86 & 42 & 100 & 100 & 100 & 100 \\
\hline $98 \%$ 吡虫啉 & 100 & 100 & 35 & 100 & 100 & 100 & 100 \\
\hline
\end{tabular}

$a$ 测试浓度为 $600 \mu \mathrm{g} / \mathrm{mL} ;^{b}$ 测试浓度为 $5 \mu \mathrm{g} / \mathrm{mL}$. 
表 5 Aspernigerin 的类似物离体杀菌活性测试结果(抑制率/\%)

Table 5 Fungicidal activity (inhibition rate/\%) in vitro of aspernigerin analogues

\begin{tabular}{|c|c|c|c|c|c|c|}
\hline Compd. & 小麦赤霉病 & 水稻纹枯病 & 黄瓜灰電病 & 番茄早疫病 & 烟草赤星病 & 黄瓜炭疽病 \\
\hline Aspernigerin & 33 & 58 & 51 & 28 & 0 & 0 \\
\hline $2 a$ & 0 & 0 & 51 & 25 & 0 & 0 \\
\hline $2 \mathbf{b}$ & 0 & 0 & 49 & 0 & 0 & 0 \\
\hline $2 c$ & 22 & 0 & 51 & 0 & 0 & 0 \\
\hline 2d & 49 & 52 & 75 & 58 & 47 & 46 \\
\hline $2 e$ & 61 & 43 & 67 & 40 & 28 & 29 \\
\hline $2 f$ & 36 & 0 & 49 & 35 & 29 & 25 \\
\hline $2 \mathrm{~g}$ & 37 & 37 & 51 & 42 & 25 & 23 \\
\hline $2 \mathrm{~h}$ & 36 & 0 & 43 & 41 & 24 & 25 \\
\hline $2 \mathbf{i}$ & 55 & 48 & 52 & 55 & 25 & 46 \\
\hline $2 \mathbf{j}$ & 32 & 35 & 75 & 58 & 33 & 50 \\
\hline $2 k$ & 42 & 39 & 62 & 36 & 24 & 27 \\
\hline 21 & 0 & 0 & 52 & 25 & 0 & 0 \\
\hline $2 \mathrm{~m}$ & 0 & 0 & 33 & 0 & 0 & 0 \\
\hline $2 n$ & 35 & 0 & 59 & 38 & 25 & 25 \\
\hline 20 & 0 & 43 & 30 & 32 & 0 & 0 \\
\hline 苯醚甲环唑 & 100 & 83 & 84 & 100 & 100 & 100 \\
\hline
\end{tabular}

构，经简单的结构改造，发现该类化合物在杀虫活性以 及杀菌活性方面都有很好表现, 此类结构化合物可能存 在良好应用前景，值得进一步研究.

\section{References}

[1] Shen, L.; Ye, Y.-H.; Wang, X.-T.; Zhu, H.-L.; Xu, C.; Song, Y.-C.; Li, H.; Tan, R.-X. Chem. Eur. J. 2006, 12, 4393.

[2] Shen, L. Ph.D. Dissertation, Nanjing University, Nanjing, 2006 (in Chinese).

(申丽, 博士论文, 南京大学, 南京, 2006.)

[3] Frank, D. P.; Ernest, C.; Robert, B. D.; Warren, K. J. Med. Chem 1962, 5, 398.

[4] Sarah, C.; Raymond, H.; Jean-Pierre, H. J. Org. Chem. 2002, 67, 3502.

[5] Chen, N.-C. Bioassay of Pesticides, Beijing Agricultural University Press, Beijing, 1991, pp. 161 162 (in Chinese).

(陈年春, 农药生物测定技术, 北京农业大学出版社, 北京, 1991, pp. 161 162.)

[6] Chong, J. A.; Fanger, C.; Larson, G. R.; Lumma, W. C.; Moran, M. M.; Ripka, A.; Underwood, J. D.; Weigele, M.; Zhen X. G. US
2007213321, 2007 [Chem. Abstr. 2007, 147, 365488].

[7] Wang, Z.-Y.; Wang, C.-Z.; Wang, F.-X.; Yang, J. Chin. J. Pharm 2005, 36, 261 (in Chinese).

(王志远，王成章，王福祥，杨洁，中国医药工业杂志，2005，36, 261.)

[8] Silverman, I. R.; Ali, S. F.; Cohen, D. H.; Lyga, J. W.; Simmons, K. A.; Cullen, T. G. WO 9726252, 1997 [Chem. Abstr. 1997, 127, 176441].

[9] Li, Z.-G.; Huang, R.-Q.; Shao, R.-L.; Ma, J.-A. Chin. J. Synth Chem. 2000, 8, 130 (in Chinese). (李在国，黄润秋，郡瑞链，马军安，合成化学，2000, 8,130 .)

[10] Beth, A. L.; James, M. R.; Thomas, C. S.; Michael, T. S.; Irene, M. M.; Jeffery, D. W. US 2007004750, 2007 [Chem. Abstr. 2007, 146, 121988].

[11] Li, G.-Y.; Yan, S.-G.; Jiang, S.; Qian, X.-H.; Huang, Q.-C.; Zhang, R. Chin. J. Org. Chem. 2008, 28, 2001. (李刚月, 阎圣刚, 蒋山, 钱旭红, 黄青春, 张蓉, 有机化学, 2008, 28, 2001.)

[12] Cai, M.-Y.; Li, Z.; Fan, F.; Huang, Q.-C.; Shao, X.-S.; Song, G.-H. J. Agric. Food Chem. 2010, 58, 2624

(Qin, X.) 